JOURNAL OF SYNCHROTRON RADIATION

ISSN $1600-5775$

Received 18 March 2019

Accepted 17 June 2019

Edited by V. Favre-Nicolin, CEA and Université Joseph Fourier, France

Keywords: multimodal imaging; X-ray diffraction imaging; scanning near-field optical microscopy; structure-property correlations; insulator-metal transitions; samarium sulfide; scanning probe microscopy.

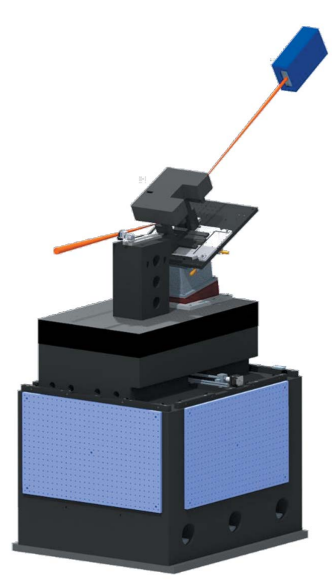

OPEN $\odot$ ACCESS

\section{Simultaneous scanning near-field optical and X-ray diffraction microscopy for correlative nanoscale structure-property characterization}

\author{
Qian Li, ${ }^{a}$ Samuel D. Marks, ${ }^{\text {a,b }}$ Sunil Bean, ${ }^{a}$ Michael Fisher, ${ }^{a}$ Donald A. Walko, \\ Anthony D. DiChiara, ${ }^{a}$ Xinzhong Chen, ${ }^{c}$ Keiichiro Imura, ${ }^{d}$ Noriaki K. Sato, ${ }^{d}$ \\ Mengkun Liu, ${ }^{\mathrm{c}, \mathrm{e}}$ Paul G. Evans ${ }^{\mathrm{b}}$ and Haidan Wen ${ }^{\mathrm{a} *}$
}

\footnotetext{
${ }^{\mathbf{a}}$ Advanced Photon Source, Argonne National Laboratory, Lemont, IL 60439, USA, ${ }^{\mathbf{b}}$ Department of Materials Science and Engineering, University of Wisconsin-Madison, Madison, WI 53706, USA, 'Department of Physics, Stony Brook University, Stony Brook, NY 11794, USA, 'Department of Physics, Nagoya University, Nagoya 464-8602, Japan, and $\mathbf{e}^{\mathbf{e}}$ Department of Physics, Southern University of Science and Technology, Shenzhen 518055, People's Republic of China. *Correspondence e-mail: wen@anl.gov
}

A multimodal imaging instrument has been developed that integrates scanning near-field optical microscopy with nanofocused synchrotron X-ray diffraction imaging. The instrument allows for the simultaneous nanoscale characterization of electronic/near-field optical properties of materials together with their crystallographic structure, facilitating the investigation of local structureproperty relationships. The design, implementation and operating procedures of this instrument are reported. The scientific capabilities are demonstrated in a proof-of-principle study of the insulator-metal phase transition in samarium sulfide (SmS) single crystals induced by applying mechanical pressure via a scanning tip. The multimodal imaging of an in situ tip-written region shows that the near-field optical reflectivity can be correlated with the heterogeneously transformed structure of the near-surface region of the crystal.

\section{Introduction}

Many functional materials exhibit structural and/or chemical heterogeneities at nano- and mesoscopic scales. The heterogeneities can arise from a variety of intrinsic and extrinsic factors. Representative systems incorporating intrinsic heterogeneities include ferroics and phase-changing materials, in which the functionality of the material is closely linked to structural or electronic heterogeneity. Ferroic systems exhibit a symmetry-lowering transition which can split the material into multiple domain variants with distinct structural distortion (Lummen et al., 2014), polar ion displacements (Li et al., 2015), magnetic spin alignments (Evans et al., 2002) and combinations thereof. Phase coexistence and separation are also commonly observed in a wider variety of systems exhibiting first-order phase transitions because of their hysteretic thermodynamics, the insulator-metal phase transition (IMT) in $\mathrm{VO}_{2}$ (Liu et al., 2013) being a canonical example. Defects such as vacancies contribute to the chemical state of materials and in many cases strongly interact with the intrinsic phase or domain stabilities (Sharma et al., 2018). Determining the spatial configurations of coexisting phases in heterogeneous materials is of considerable importance for understanding the ensemble-averaged properties of these materials. Experimental understanding can be obtained via nanoscale imaging of electronic, optical, thermal and magnetic heterogeneities, and the response of heterogeneities to external fields and optical transients. Ultimately, comprehensive insight into 
these heterogeneities and their dynamics provides the means to control and manipulate the corresponding functionalities.

Advances in synchrotron-radiation technologies have led to a rapid series of recent developments in X-ray diffraction microscopy (XDM) (Sakdinawat \& Attwood, 2010; Cornelius \& Thomas, 2018; Wen et al., 2018). XDM is based on a darkfield imaging mechanism and thus yields quantitative information about the spatial variations in crystallographic aspects of materials such as strain and lattice tilt, with lateral spatial resolution reaching sub-100 nm length scales (Holt et al., 2013; Laanait et al., 2014). XDM requires minimal non-destructive sample preparation and allows for the coupling of complex sample environments with in situ structural characterization. A complementary family of surface-imaging tools based on scanning-probe microscopy (SPM) has various modalities that probe specific aspects of material properties corresponding to their functional response. Combined SPM and XDM beamline instruments have been developed (Rodrigues et al., 2009; Ren et al., 2014; Vitorino et al., 2016) in order to take advantage of in situ imaging capabilities. These instruments are commonly based on a scanning nanofocused X-ray beam scheme interfaced with a compact home-built SPM apparatus using specific detection techniques, such as tuning forks (Rodrigues et al., 2009) or self-sensing probes (Ren et al., 2014). Studies conducted with these instruments have included the structural characterization of nanostructures subjected to nanomechanical loading via SPM tips or the morphological evaluation of $\mathrm{X}$-ray beam-induced damages in soft materials (Vitorino et al., 2016). For example, coherent $X$-ray diffraction patterns were recorded from individual $\mathrm{Au}$ nanoislands during indentation (Dupraz et al., 2017) providing microscopic details about the material-deformation processes. However, these beamline SPM instruments only provide limited imaging modalities, such as topography, while important nanoscale functional properties, such as local electronic and optical heterogeneities, cannot be simultaneously characterized.

As a standalone technique, SPM can provide only indirect structural information and is thus often inadequate in ascertaining structure-property correlations. Scattering-type scanning near-field optical microscopy (s-SNOM), for example, can probe near-field optical properties within a broad spectral range from terahertz to visible light, enclosing the response of electronic charge carriers, phonons and other collective excitations in the materials (Atkin et al., 2012). Combining s-SNOM with XDM yields a new multimodal imaging platform that is capable of studying nanoscale structure-property relationships for a diverse set of materials systems. Although ex situ measurements have provided nanoscale mapping during IMTs in correlated systems (Zhang et al., 2019; Liu et al., 2016), they are inherently subject to ambiguities in sampleenvironment calibration, scan registration and other experimental details. These ambiguities can be avoided by simultaneous imaging of electronic and structural properties using a structural probe.

In this work, we report the development of an integrated imaging platform combining synchrotron X-ray nanodiffraction imaging with SNOM. This new instrument is termed
'XSNOM' and enables correlative multimodal imaging of the crystal structure and near-field optical properties of materials at mesoscales. As demonstrated here, the XSNOM can be further extended by incorporating a general-purpose SPM, which will allow for characterizing a wide range of nanoscale material properties. In order to illustrate the potential of this approach, we have examined the local tip pressure-induced IMT in samarium sulfide ( $\mathrm{SmS}$ ) single crystals. Our results show that the near-field mid-infrared (mid-IR) optical reflectivity of SmS can be quantitatively correlated with the nearsurface crystal structure involving an intermediate, heterogeneous two-phase configuration.

\section{Experiment}

\subsection{XSNOM instrument}

The instrument was built at the 7-ID-C beamline of the Advanced Photon Source at the Argonne National Laboratory (Walko et al., 2016). A schematic and illustrative photographs of the XSNOM are presented in Fig. 1 and Fig. 2, respectively. X-ray photons with an energy of $11.5 \mathrm{keV}$ and a flux of $\sim 1 \times 10^{12}$ photons s $^{-1}$ were generated from a $2.4 \mathrm{~m}$ long undulator and monochromated using a two-crystal $\mathrm{Si}(111)$ monochromator. The X-ray beam passes through a series of diagnostic and control devices, including slits, filters and ion chambers, before reaching the focusing optical system that consists of a gold beam stop, a gold Fresnel zone plate (FZP) and an order-sorting aperture (OSA). The FZP has a diameter of $320 \mu \mathrm{m}$ and an outermost-zone width of $80 \mathrm{~nm}$, and images the X-ray source onto the focal plane with a beam size of $350 \mathrm{~nm}$ (vertical) $\times 1000 \mathrm{~nm}$ (horizontal) at a working distance of $237 \mathrm{~mm}$ at $11.5 \mathrm{keV}$. The FZP and beam stop are assembled in a manually adjustable holder, and the whole assembly is mounted on a piezomotor $x y z$ stage (Smaract $\mathrm{GmbH}$ ) that has better than $30 \mathrm{~nm}$ position repeatability in closed-loop control. A separate step motor-driven $x y z$ stage is used to position the OSA because of its less stringent precision

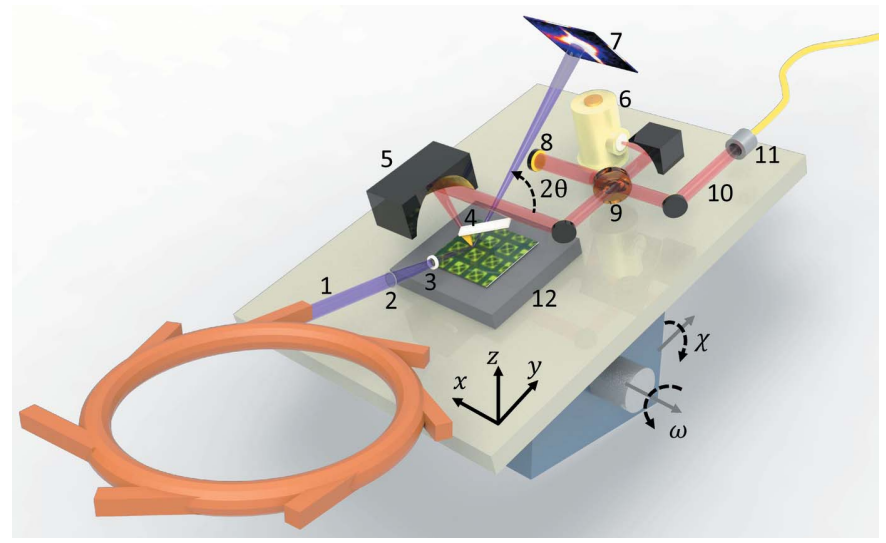

Figure 1

Schematic of the XSNOM. The main components are (1) synchrotron X-ray beam, (2) FZP, (3) OSA, (4) probe tip, (5) parabolic mirror, (6) mid-IR detector, (7) X-ray area detector, (8) vibrating mirror, (9) beam splitter, (10) mid-IR laser beam, (11) optical fiber and (12) $x y z$ piezo scanner. 


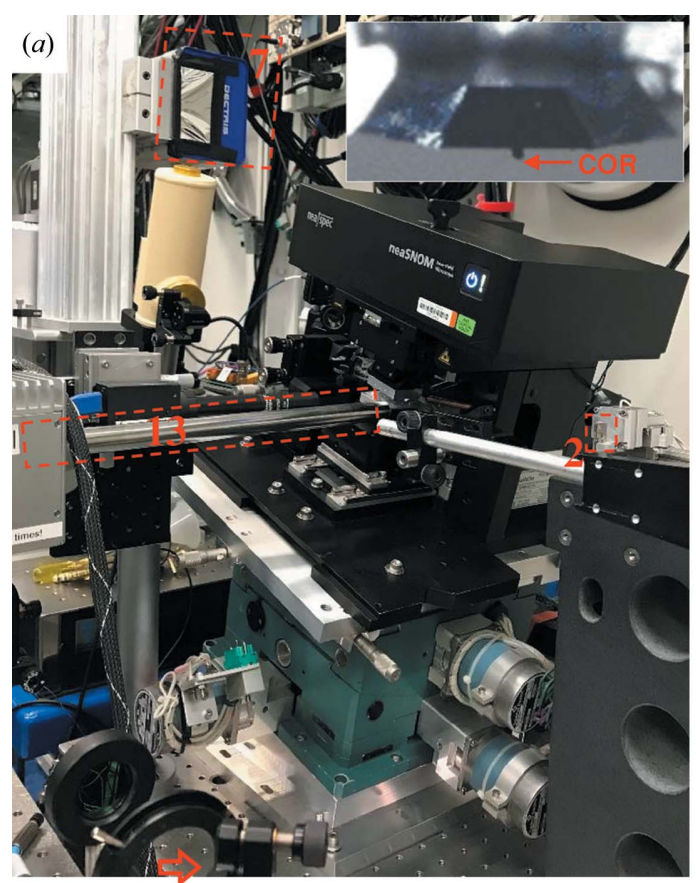

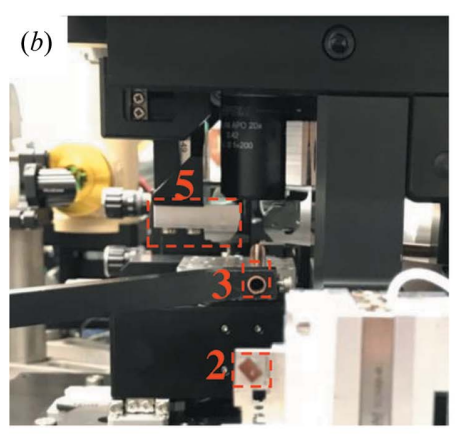

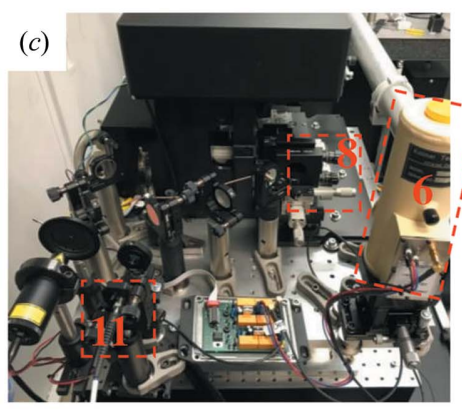

Figure 2

(a) Photograph taken at the tilted working condition. The arrow at the lower left denotes the output of the $\mathrm{CO}_{2}$ laser. Inset is a front-camera view of the probe tip, with the arrow denoting the COR. Photographs of $(b)$ the X-ray focusing optics and $(c)$ the mid-IR optics, looking downstream and upstream, respectively, from the opposite sides of the SNOM. Components (1)-(12) are numbered as in Fig. 1. Component (13) is the X-ray fluorescence detector.

requirements. The FZP and OSA stages are moved together to scan the position of the focused X-ray beam. Within the typical tens-of-micrometres coarse alignment range required of the FZP, the movement of the focusing optics does not affect the focused beam size or flux.

The main component of the XSNOM is a commercial scanning near-field optical microscope (Neaspec SNOM): a sample-scanning, optical beam deflection type of SPM with sufficient open space around the sample stage to allow the passage of the incident and diffracted X-ray beams. The microscope has a rigid design which allows operation under tilted conditions without sacrificing its performance (up to $30^{\circ}$ as tested). The SNOM is mounted on a Huber two-circle $\left(0-30^{\circ}\right.$ in $\omega$ and $\pm 10^{\circ}$ in $\left.\chi\right)$ goniometer interfaced with an $\mathrm{Al}$ adapter plate that extends on one side to hold the SNOM optical components. The $20 \times$ optical microscope objective lens [see Fig. 2(b)] of the SNOM provides a convenient $z$-axis reference point; the cantilevers of probes are aligned onto the focal plane of the deflection laser beam to maintain a constant height of the tip-sample junction. Minor variations, e.g. in cantilever thickness and tip height, remain and may be compensated for during the alignment process. The tipsample junction is adjusted to coincide with the center-ofrotation (COR) of the goniometer so that a sample regionof-interest (ROI) moves by a negligible amount $(<200 \mathrm{~nm})$ relative to the focal spot of the X-ray beam during typical rocking scans spanning an angular range $\Delta \omega$ of $1-2^{\circ}$. The alignment was optimized by carefully adjusting the SNOM positions by translating the $\mathrm{Al}$ adapter plate. The sphere of confusion was measured to be less than $10 \mu \mathrm{m}$ over the full $30^{\circ} \omega$-rotation range using a high-resolution video camera. A corresponding still image is inset in Fig. 2(a).

The s-SNOM instrument measures the light locally scattered from a nearfield coupled metallic SPM tip-sample junction. The characteristic length scale of the measurement is on the order of the tip size, typically tens of nanometres, which is much smaller than the wavelength of the light (Berweger et al., 2012). A pseudo-heterodyne detection scheme with stringent optical alignment is implemented to remove far-field background from the near-field signals (Deutsch et al., 2008). The standard SNOM optical setup was modified to allow the SNOM to be positioned at various tilts for XDM. The mid-IR (wavelengths of $9.2-10.7 \mu \mathrm{m}$ ) probing light is generated from a $\mathrm{CO}_{2}$ laser (L4S, Access Laser). The size of the laser prevents its direct placement on the goniometer with the microscope because the imbalanced weight distribution on the goniometer would significantly reduce the stability of the combined instrument. The laser was thus mounted on a separate platform. This separation raises significant issues about free-space laser-beam alignment since the XSNOM operates under varying tilted conditions as opposed to the stationary configuration it would conventionally have on a horizontal optical table with fixed overall orientation. To circumvent the potential misalignment caused by tilting, a $2 \mathrm{~m}$-long hollow-core mid-IR fiber is used to couple the laser output into the SNOM optical components on the goniometer. The fiber provides the flexibility for the goniometer operation as well as the stable coupling required for SNOM measurements. The coupling lenses, mirrors and mid-IR detector are arranged in a compact layout occupying an area of 6 inch $\times 12$ inch beside the SNOM on the adapter plater [see Fig. 2(c)].

Diffracted X-ray photons were detected using a photoncounting area detector (Pilatus 100K, Dectris). The detector was mounted on a motorized two-linear-axis $(y z)$ stage at an adjustable distance of $300 \mathrm{~mm}$ to $700 \mathrm{~mm}$ from the SPM tip. In practice, values of the scattering angles $2 \theta$ are converted to the elevations in order to select the position of the detector. An energy-discriminating silicon drift detector (Vortex 60EX, Hitachi) was installed close to the sample stage at right angles to the primary X-ray beam [(13) in Fig. 2(a)] to capture fluorescence X-ray photons from the samples or SPM probes. In addition, a digital video camera coupled with a YAG scintillator crystal was placed downstream to view the transmitted X-ray beam, facilitating the alignment of multiple components of the XSNOM. 


\subsection{SPM tip and X-ray beam coalignment}

The two scanning probes, the SPM tip and the focused $\mathrm{X}$-ray beam, need to be spatially coincident in nanoscale imaging in order to create scanning maps that can be spatially registered with respect to each other. The first step in the operation of the XSNOM is the coalignment of the focused $\mathrm{X}$-ray beam and the SPM probe. This is conducted in a series of steps using various imaging-contrast mechanisms, as illustrated in Fig. 3. First, the FZP and beam stop are manually steered in the $y z$-coordinate plane to place their centers at the same place in the $y z$ plane as the tip apex of the SPM probe, based on the X-ray shadow image of these components as monitored from the downstream X-ray scintillator. The shadow image is only visible at the non-tilted condition corresponding to $\omega=0$ as in Fig. 3(a). This alignment step typically achieves uncertainties within $\pm 10 \mu \mathrm{m}$ along both directions, which serves as a spatial range for subsequent finer alignment. Next, fluorescence X-ray signals of the tip can be mapped by scanning the focused primary X-ray beam across the tip. The probe (Nanosensors model PPP-EFM) used in the experiments presented here includes a conductive coating containing a thin layer of $\mathrm{Ti}$, producing a well defined, projected tip profile consistent with its real pyramid shape [Fig. 3(b)]. The tip-apex coordinates can be readily identified with an accuracy of $\sim 1 \mu \mathrm{m}$ from such fluorescence images. Because of the imperfect COR alignment, a final tip-mapping measurement is performed at rocking angles close to the diffraction conditions of specific samples.

To achieve higher accuracy of the overlap between focused $\mathrm{X}$-ray and SPM tip, the X-ray beam alignment is further refined against samples with known features such as the $\mathrm{Au}$ antenna pattern shown in Fig. 3(c). Figs. 3(d)-3(f) show the surface topography, $\mathrm{V} K_{\alpha / \beta}$ fluorescence and $\mathrm{Au}$ diffraction intensity images for a patterned $\mathrm{Au} / \mathrm{VO}_{2}$ thin-film sample shown in the optical image in Fig. 3(c). All of these signals were simultaneously recorded while the sample was raster scanned with respect to the focused X-ray beam and the SPM tip. The images in Figs. 3(d)-3(f) provide discernable and complementary location information, and, in conjunction with SNOM channels, allow large flexibilities for the calibration procedure. A misalignment of $\sim 2 \mu \mathrm{m}$ along the $x$ direction can be precisely corrected by translating the height of the FZP by the projection of this distance along the $z$ direction, multiplying the required apparent translation by a factor of $\sin (\omega)$. By iterating this procedure, the overlap of the two probes can be achieved within the accuracy of the X-ray beam size ( 350 nm $\times 1000 \mathrm{~nm})$.

\subsection{Correlated structure/property imaging in $\mathrm{SmS}$}

The nanoscale optical reflectivity and lattice structure of surface-engineered SmS crystals were characterized by in situ SNOM and XDM measurements in order to demonstrate the capabilities of the XSNOM imaging instrument. Single-crystal SmS specimens grown by the Bridgeman method and characterized elsewhere (Gilbert Corder et al., 2017) were used in this study. The cleaved surface of the crystal was close to the [001] orientation and exhibited a dark blue luster characteristic of the insulating phase. The crystals were attached on the sample stage and aligned to the 002 specular reflection conditions. SNOM was performed in the non-contact imaging mode with a tip-specific modulation frequency of $\sim 183 \mathrm{kHz}$, and the near-field signals were demodulated at the second harmonic frequencies (i.e. the $\mathrm{S}_{2}$ channel). At fixed tilting angles, the samples were scanned along the in-plane directions of the sample surface with respect to the aligned AFM tip and focused X-ray beam. X-ray diffraction patterns were acquired at each sample position using a counting time of $100 \mathrm{~ms}$ while SNOM signals were recorded during the initial $10 \mathrm{~ms}$. To induce local patterns with increased metallicity, tip pressure was applied to the sample surface in contact scanning mode with a loading force of $2-3 \mu \mathrm{N}$. A peak pressure of several GPa near the uppermost $\sim 10 \mathrm{~nm}$ surface region was estimated by the Hertzian contact model ( $\mathrm{Li}$ et al., 2015).

\section{Result and discussion}

SmS crystals exhibit a semiconducting phase due to the localized $4 f$ electronic state of $\mathrm{Sm}^{2+}$ under ambient conditions. With increasing hydrostatic pressure, SmS evolves into a semimetal with an intermediate $\mathrm{Sm}^{2.6-2.8+}$ valence state at $\sim 6.5 \mathrm{kPa}$ and then to a metal with a $\mathrm{Sm}^{3+}$ valence state above

Figure 3

(a) Downstream X-ray projection view at the horizontal position $\left(\omega=0^{\circ}\right)$ showing the probe tip (1), beam stop (2) and FZP (3). The probe tip was aligned such that its apex fell at the center of the zone plate/beam stop. (b) Ti $K$ fluorescence intensity map of the tip apex measured at a tilted position $\sim 16^{\circ}$ from the SNOM. $(c-f)$ Multimodal views of a $\mathrm{VO}_{2}$ thin-film sample with patterned Au top electrodes: $(c)$ optical microscopy, $(d)$ AFM topography, $(e) \mathrm{V} K_{\alpha / \beta}$ fluorescence intensity and $(f) \mathrm{Au}$ 111 diffraction intensity (powder ring-integrated) images. The parts $(d)-(f)$ were acquired in a single imaging scan. 
$20 \mathrm{kPa}$. The electronic transformation is accompanied by an isostructural transition with $\sim 13.5 \%$ volumetric contraction while maintaining the NaCl-type crystal structure (Deen et al., 2005). In a recent study, SPM tip pressure was shown to robustly induce a metallic phase on the surface of SmS crystals, verified by SNOM (Gilbert Corder et al., 2017). This study illustrated the feasibility of using SPM writing techniques to pattern metastructures with a tailored optical response. However, the pressure exerted by an SPM tip can be nonuniform and confined to small sample volumes. As a result, the written regions may contain structural and electronic heterogeneities. The XSNOM can be a particularly suitable tool in gaining new insight into the nanoscale structure-property relationship of pressure-transformed materials such as SmS.

Fig. 4(a) presents an X-ray area-detector image of the diffracted X-ray beam captured at $9.8^{\circ}$ incident angle within an in situ tip-written region of SmS. The X-ray penetration depth $(\sim 1.5 \mu \mathrm{m})$ allows measurements of both the induced phase at the surface and the underlying pristine phase. Since the Bragg peak width of the induced phase is sufficiently wide, the diffraction peaks from both phases can be observed simultaneously in distinct regions-of-interest from a single incident angle. The lowerscattering angle reflection (ROI 1) corresponds to the pristine phase, while that of the induced phase appears at the $2 \theta$ angle $1.93^{\circ}$ higher (ROI 2). Apart from these two reflections, no isolated peaks have been observed throughout our study. Based on the observed angles, the induced phase has a smaller lattice parameter of $c=$ $5.69 \AA$ while the pristine phase has a lattice parameter of $5.97 \AA$. This difference in the lattice parameters is consistent with previous structural studies (Sousanis et al., 2016). A set of rocking curves at specific single locations were measured at the center of the written region such that the X-ray beam shift was kept within written regions. As shown in Fig. 4(b), the incident angles of the Bragg reflections of the two phases differ by $\sim 1^{\circ}$, half of the difference in the $2 \theta$ angles of the reflections. Overall, the induced phase thus has the same lattice orientation as the pristine phase, as would be expected if the transformation proceeds via a mechanism in which the crystallographic axes of the induced and pristine phases are aligned at their interface. The rocking width of the induced phase is nearly twice that of the pristine phase indicating that the induced phase has a larger mosaicity than the pristine crystal. A similar difference in the angular widths of the reflections is apparent in the $\chi$ angular direction in Fig. 4(a).

Simultaneous electronic and lattice structural imaging were performed over the $10 \mu \mathrm{m} \times 10 \mu \mathrm{m}$ written region, as presented in Figs. 5(a) and 5(b). Both the SNOM and XDM images exhibit irregular patterns, indicating that the tip pressure during the writing process is non-uniform presumably because of tip wear. The resulting state contains electronically untransformed and transformed regions, which exhibit multiple levels of contrast in the total diffraction intensity. The metallic nature of the induced phase is confirmed by its strong mid-IR (wavelength $\simeq 10 \mu \mathrm{m}$ ) near-field signals originating from the Drude-like reflectivity response of conducting electrons. Note that the SNOM image obtained here is undersampled because the step size of the sample scan was chosen to match the X-ray beam width, an order of magnitude larger than the lateral resolution of the SNOM.

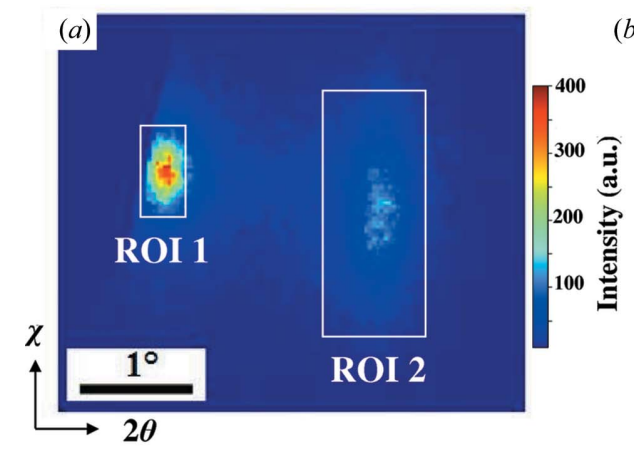

Figure 4

(a) X-ray detector image of the 002 Bragg peaks of SmS measured at $\omega=9.8^{\circ}$, showing the characteristic reflections from the pristine phase (ROI 1) and the induced phase (ROI 2) appearing at this incident angle. (b) Rocking curves of both pristine and induced phases with their ROIintegrated diffraction intensities recorded from a series of detector images measured as a function of $\omega$ angle.
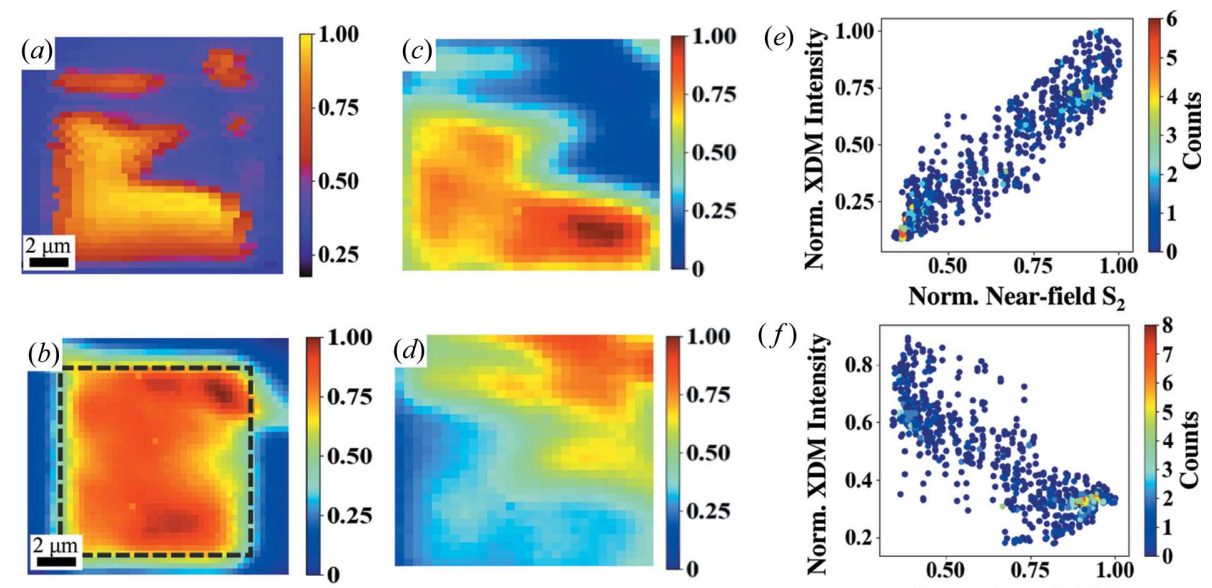

Figure 5

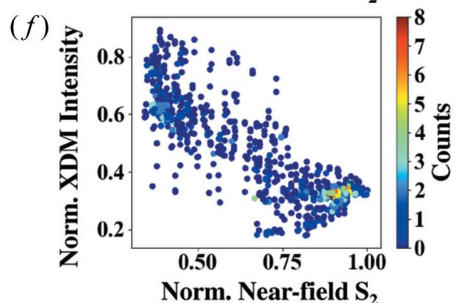

(a) Mid-IR near-field scattering amplitude $\left(\mathrm{S}_{2}\right)$ and $(b)$ total X-ray diffraction intensity from the tippressure-written pattern in SmS. The region within the dashed box is separated into X-ray diffraction contributions from $(c)$ the induced phase and $(d)$ the pristine phase, and correlated to the corresponding section of the SNOM map in $(a)$. The color bars in $(a-d)$ show the normalized intensity. A positive correlation $(e)$ exists between the SNOM amplitude and the X-ray intensity from the induced phase, while a corresponding negative correlation $(f)$ arises from the untransformed region. 
To further reveal correlations between the observed structural and electronic heterogeneities, we decompose the XDM intensity into the contributions from the metallic [Fig. 5(c)] and insulating phases [Fig. 5(d)] using the ROI-integration scheme as depicted in Fig. 4(a). The resultant structural contrast maps are each correlated with the corresponding SNOM near-field image [Fig. 5(a)] generating a pixel-wise joint histogram. The histogram for the metallic phase indicates a strong, evidently linear correlation within the entire signal ranges [Fig. 5(e)]; correspondingly, an apparently negative correlation is found for the insulating phase [Fig. 5(f)]. Within the kinematic approximation of diffraction, the XDM intensities are proportional to the fractional compositions of both of the SmS phases and thus reflect their depth profiles at each lateral position. The near-field optical response is associated with the carrier density in the metallic SmS phase layer, which in turn determines the penetration depth of the incident midIR photons (Chen et al., 2017; Liu et al., 2016). The observation of linear correlation suggests that the IMT phase boundary is located at a depth that is still within the probingdepth range of SNOM; otherwise, a very steep large-slope trend would appear in the correlation plot because the nearfield optical response no longer increases with the thickness of the metallic layer. With more prior knowledge of samples (as frequently encountered in thin films with a known thickness smaller than X-ray penetration depths), the ability to assess structural configurations using XDM can inform the modeling of near-field optical response, thereby providing quantitative information for correlating local lattice and electronic properties.

\section{Conclusion}

$\mathrm{XSNOM}$ is a new hard X-ray instrument combining s-SNOM with X-ray diffraction microscopy. An effective probe-alignment protocol and the experimental procedures were established by showcasing the concurrent measurements of the electronic and lattice structural response of strain-engineered surfaces of SmS single crystals. XSNOM brings new scientific capabilities for multimodal correlative imaging of material crystal structures and functional properties as well as in situ local manipulation of materials through a SPM tip. New microscopic insights can be unambiguously gained by correlating nanoscale electronic and structural properties. We anticipate that the upgrade of the Advanced Photon Source with a low-emittance electron storage ring and significantly enhanced coherence will further benefit the X-ray diffractionimaging capabilities by allowing the use of higher numerical aperture X-ray optics while preserving the X-ray intensity and incorporation of coherent-imaging techniques such as ptychography (Pfeiffer, 2018); both approaches may improve the XDM spatial resolution to match the resolution of SNOM. Apart from s-SNOM, it is also feasible to implement other SPM modalities, such as piezoresponse force microscopy and magnetic force microscopy, on the existing platform with reasonable efforts. Therefore, XSNOM can be tailored for investigating structure-property relationships in a wide range of systems including multiferroics, energy materials and strongly correlated quantum materials.

\section{Acknowledgements}

The authors would like to thank Dr Michael J. Wojcik and Dr Curt A. Preissner for the design and fabrication of zone plate and holder.

\section{Funding information}

HW and QL were supported by the US Department of Energy (DOE), Office of Science, Materials Science and Engineering Division. SM and PGE acknowledge support from the US DOE, Office of Science, Basic Energy Sciences under Contract No. DE-FG02-04ER46147. This research used resources of the Advanced Photon Source and Center for Nanoscale Materials, a US DOE Office of Science User Facility operated for the DOE Office of Science by the Argonne National Laboratory under Contract No. DE-AC0206CH11357. This material is based upon work supported by the US DOE, Office of Science, Office of Workforce Development for Teachers and Scientists, Office of Science Graduate Student Research (SCGSR) program. The SCGSR program is administered by the Oak Ridge Institute for Science and Education for the DOE under contract number DE-SC0014664.

\section{References}

Atkin, J. M., Berweger, S., Jones, A. C. \& Raschke, M. B. (2012). Adv. Phys. 61, 745-842.

Berweger, S., Atkin, J. M., Olmon, R. L. \& Raschke, M. B. (2012). J. Phys. Chem. Lett. 3, 945-952.

Chen, X., Lo, C. F. B., Zheng, W., Hu, H., Dai, Q. \& Liu, M. (2017). Appl. Phys. Lett. 111, 223110.

Cornelius, T. W. \& Thomas, O. (2018). Prog. Mater. Sci. 94, 384434.

Deen, P. P., Braithwaite, D., Kernavanois, N., Paolasini, L., Raymond, S., Barla, A., Lapertot, G. \& Sanchez, J. P. (2005). Phys. Rev. B, 71, 245118.

Deutsch, B., Hillenbrand, R. \& Novotny, L. (2008). Opt. Express, 16, 494-501.

Dupraz, M., Beutier, G., Cornelius, T. W., Parry, G., Ren, Z., Labat, S., Richard, M. I., Chahine, G. A., Kovalenko, O., De Boissieu, M., Rabkin, E., Verdier, M. \& Thomas, O. (2017). Nano Lett. 17, 6696-6701.

Evans, P. G., Isaacs, E. D., Aeppli, G., Cai, Z. \& Lai, B. (2002). Science, 295, 1042-1045.

Gilbert Corder, S. N., Chen, X., Zhang, S., Hu, F., Zhang, J., Luan, Y., Logan, J. A., Ciavatti, T., Bechtel, H. A., Martin, M. C., Aronson, M., Suzuki, H. S., Kimura, S., Iizuka, T., Fei, Z., Imura, K., Sato, N. K., Tao, T. H. \& Liu, M. (2017). Nat. Commun. 8, 2262.

Holt, M., Harder, R., Winarski, R. \& Rose, V. (2013). Annu. Rev. Mater. Res. 43, 183-211.

Laanait, N., Zhang, Z., Schlepütz, C. M., Vila-Comamala, J., Highland, M. J. \& Fenter, P. (2014). J. Synchrotron Rad. 21, $1252-1261$.

Li, Q., Cao, Y., Yu, P., Vasudevan, R., Laanait, N., Tselev, A., Xue, F., Chen, L., Maksymovych, P., Kalinin, S. \& Balke, N. (2015). Nat. Commun. 6, 8985.

Liu, M., Sternbach, A. J. \& Basov, D. N. (2016). Rep. Prog. Phys. 80, 014501 . 
Liu, M. K., Wagner, M., Abreu, E., Kittiwatanakul, S., McLeod, A., Fei, Z., Goldflam, M., Dai, S., Fogler, M. M., Lu, J., Wolf, S. A., Averitt, R. D. \& Basov, D. N. (2013). Phys. Rev. Lett. 111, 096602.

Lummen, T. T. A., Gu, Y., Wang, J., Lei, S., Xue, F., Kumar, A., Barnes, A. T., Barnes, E., Denev, S., Belianinov, A., Holt, M., Morozovska, A. N., Kalinin, S. V., Chen, L.-Q. \& Gopalan, V. (2014). Nat. Commun. 5, 3172.

Pfeiffer, F. (2018). Nat. Photon. 12, 9-17.

Ren, Z., Mastropietro, F., Davydok, A., Langlais, S., Richard, M.-I., Furter, J.-J., Thomas, O., Dupraz, M., Verdier, M., Beutier, G., Boesecke, P. \& Cornelius, T. W. (2014). J. Synchrotron Rad. 21, $1128-1133$.

Rodrigues, M. S., Cornelius, T. W., Scheler, T., Mocuta, C., Malachias, A., Magalhães-Paniago, R., Dhez, O., Comin, F., Metzger, T. H. \& Chevrier, J. (2009). J. Appl. Phys. 106, 103525.

Sakdinawat, A. \& Attwood, D. (2010). Nat. Photon. 4, 840-848.

Sharma, Y., Balachandran, J., Sohn, C., Krogel, J. T., Ganesh, P., Collins, L., Ievlev, A. V., Li, Q., Gao, X., Balke, N., Ovchinnikova,
O. S., Kalinin, S. V., Heinonen, O. \& Lee, H. N. (2018). ACS Nano, 12, 7159-7166.

Sousanis, A., Smet, P. F., Detavernier, C. \& Poelman, D. (2016). IEEE Nanotechnology Materials and Devices Conference (NMDC), 9-12 October 2016, Toulouse, France, pp. 1-2. IEEE Xplore.

Vitorino, M. V., Fuchs, Y., Dane, T., Rodrigues, M. S., Rosenthal, M., Panzarella, A., Bernard, P., Hignette, O., Dupuy, L., Burghammer, M. \& Costa, L. (2016). J. Synchrotron Rad. 23, 1110-1117.

Walko, D. A., Adams, B. W., Doumy, G., Dufresne, E. M., Li, Y., March, A. M., Sandy, A. R., Wang, J., Wen, H. \& Zhu, Y. (2016). AIP Conf. Proc. 1741, 030048.

Wen, H., Cherukara, M. J. \& Holt, M. V. (2018). arXiv:1811.03785.

Zhang, J., McLeod, A. S., Han, Q., Chen, X., Bechtel, H. A., Yao, Z., Gilbert Corder, S. N., Ciavatti, T., Tao, T. H., Aronson, M., Carr, G. L., Martin, M. C., Sow, C., Yonezawa, S., Nakamura, F., Terasaki, I., Basov, D. N., Millis, A. J., Maeno, Y. \& Liu, M. (2019). Phys. Rev. $X, \mathbf{9}, 011032$. 